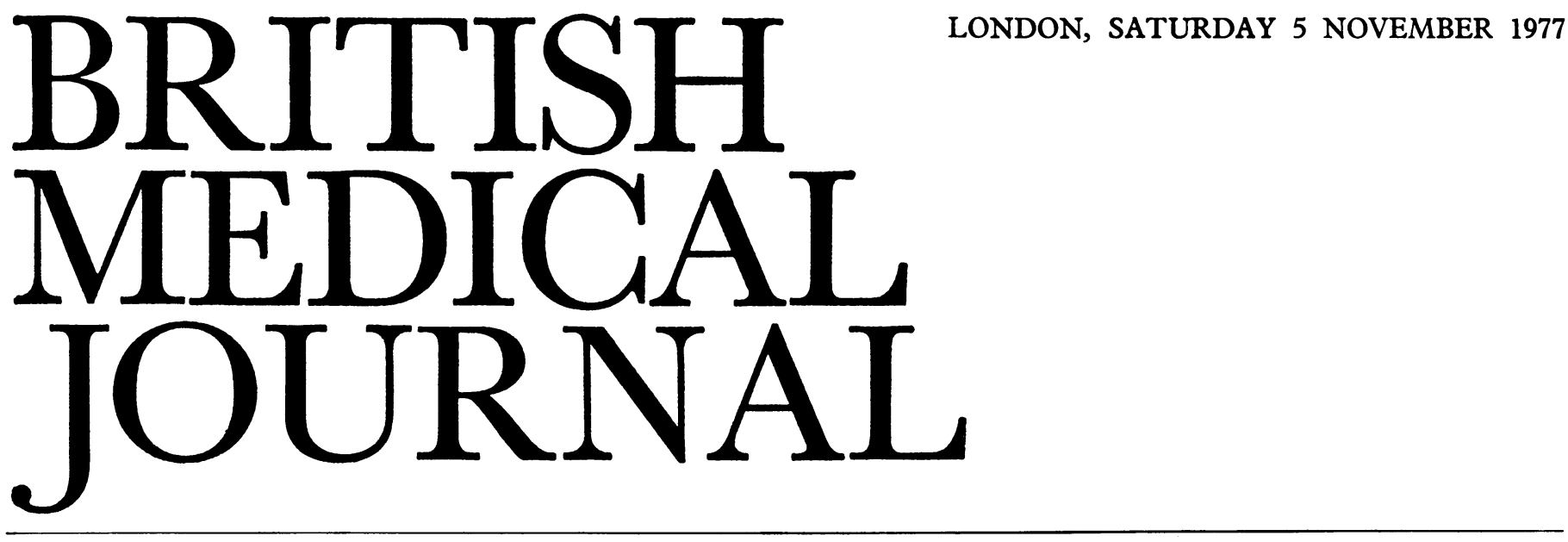

\title{
Chronic hepatitis
}

Accurate diagnosis is built on careful definitions, which may explain why active chronic (or chronic active) hepatitis seems to cause more confusion than other liver diseases. Use of the term is gradually changing, but chronic hepatitis is now best defined as an inflammation of the liver continuing for at least three to six months, as assessed from the length of symptoms or by liver function tests-though this arbitrary definition requires that overnight a prolonged acute hepatitis transforms into chronic hepatitis. In 1968 a meeting of 11 pathologists agreed to divide the histological appearances of chronic hepatitis into aggressive (which often progresses to cirrhosis) and persistent (or benign) patterns. ${ }^{1}$ This they did by assessing the degree of fibrosis and of infiltration of lobules by inflammatory cells around portal tracts. The classification has proved useful, for most biopsy specimens from patients with the clinical syndrome of active chronic hepatitis-that is, with perhaps jaundice, hepatosplenomegaly, spider naevi, and greatly raised serum concentrations of the transaminaseshave the appearances of aggressive rather than persistent hepatitis.

In most patients with active chronic hepatitis the cause is unknown, though it is plausible to attribute the condition to an altered, perhaps depressed, immunological reaction to an initial infection with a hepatitis virus. ${ }^{2}$ In Britain $10-20 \%$ of the patients are carriers of hepatitis B surface antigen ( $\mathrm{HBsAg}$ ) in their blood, while many other patients give a history of what sounds like an initial episode of viral hepatitis. There is also a high incidence of antibodies to smooth muscle and nucleoprotein and of raised immunoglobulin concentrations. In a few patients more specific causes can be identified: drugs, such as methyldopa, ${ }^{3}$ nitrofurantoin, ${ }^{4}$ and the laxative oxyphenisatin $^{5}$; Wilson's disease ${ }^{6}$; and homozygous alpha ${ }_{1}$-antitrypsin deficiency. ${ }^{7}$ The histological appearances may be mimicked by alcoholic liver disease-often difficult to exclude-and primary biliary cirrhosis may also confuse the diagnosis.

Clearly it would be helpful to know in advance which few of the many patients with acute viral hepatitis will later progress to active chronic hepatitis. The clues have recently been reviewed ${ }^{8}$ but are of limited value. In the blood of some patients who carry HBsAg there is another antigen, e antigen, which is found most often in those progressing towards (or who have developed) active chronic hepatitis. ${ }^{9}$ After an attack of hepatitis the most reasonable policy seems to be simple observation of the liver function tests; if these remain -clearly abnormal a liver biopsy should be performed three to six months after the first symptom. Patients with no history of hepatitis who are found to have abnormal function test values by chance should also be followed up and investigated if the abnormalities persist for over three to six months.

The prognosis of patients with florid active chronic hepatitis is not good, as was shown in a recent report ${ }^{10}$ of 85 patients, 26 of whom died during a median time of observation of almost four years. We now have good evidence, however, initially from Copenhagen in $1969^{11}$ and later from careful trials in London ${ }^{12}{ }^{13}$ and at the Mayo Clinic, ${ }^{14} 15$ that in adequate doses corticosteroid treatment strikingly increases wellbeing, improves liver function, and may change the histological appearances of the liver to those of chronic persistent hepatitis or to normal. The large continuing trial at the Mayo Clinic, which was led by the late W H J Summerskill, ${ }^{15}$ has shown that either a large daily dose of prednisone or a small dose combined with azathioprine can be effective, but that alternate-day doses may not be as good. Some patients seem to be cured, but follow-up is still relatively short.

It is difficult, however, to write a clear schedule for treating patients, if only because the results of the trials are not easy to compare. The severity of the disease and the patients' length of history before the trial have varied. HBsAg-negative patients have chiefly been studied, and for them it is reasonable to control the disease with $20-60 \mathrm{mg}$ prednisolone daily, reducing this over two to four months to a maintenance dose of 10-20 mg daily for at least six to 12 months. If test results are then normal a repeat liver biopsy specimen should be obtained and if the histological appearances are normal, or show only persistent hepatitis, steroids may be withdrawn slowly. If relapse follows-and this may be delayed for up to 18 months-then a further full course should be given. If steroid side effects occur, or if the biochemical values remain very abnormal on adequate doses, then azathioprine $50-100 \mathrm{mg}$ daily should be added.

Whether such treatment benefits patients with active chronic hepatitis who carry HBsAg remains doubtful, for they may have a different disease. ${ }^{16}$ Those with florid disease who were included in the trials have done well, but in general HBsAg carriers have less active disease than non-carriers and may not benefit from treatment at all. At present it seems reasonable to treat those carriers who have jaundice or whose transaminase concentrations are, say, four times the normal value, but to withhold treatment from and follow up those with less active 
disease. Trials are in progress and the results should resolve some of these uncertainties.

1 De Groote, J, et al, Lancet, 1968, 2, 626.

2 Eddleston, A L W F, and Williams, R, Lancet, 1974, 2, 1543.

3 Toghill, P J, et al, British Medical fournal, 1974, 3, 545.

- Klemola, H, et al, Scandinavian fournal of Gastroenterology, 1975, 10, 501.

${ }^{5}$ Dietrichson, O, et al, Scandinavian fournal of Gastroenterology, 1974, 9, 473.

6 Sternlieb, I, and Scheinberg, I H, Annals of Internal Medicine, 1972, 76, 59.

${ }^{7}$ Brunt, P W, Gut, 1974, 15, 573.

Sherlock, S, Lancet, 1976, 2, 354

${ }^{9}$ Eleftheriou, $\mathrm{N}$, et al, Lancet, 1975, 2, 1171.

10 Dietrichson, O, and Christoffersen, P, Scandinavian fournal of Gastroenterology, 1977, 12, 289.

11 Copenhagen Study Group for Liver Diseases, Lancet, 1969, 1, 119.

12 Cook, G C, Mulligan, R, and Sherlock, S, Quarterly fournal of Medicine, 1971, 40, 159.

13 Murray-Lyon, I M, Stern, R B, and Williams, R, Lancet, 1973, 1, 735.

14 Soloway, R D, et al, Gastroenterology, 1972, 63, 820.

15 Summerskill, W H J, et al, Gut, 1975, 16, 876.

16 Berk, P D, et al, Annals of Internal Medicine, 1976, 85, 523.

\section{Donor pretreatment}

Can treatment given to a kidney donor before removal of the organ improve the chances of transplant survival? Several transplantation teams believe that it can.

The background to this new approach is the variability in the distribution of transplantation antigens-the genetically determined constituents of the cell surface which characterise the individuality of the organism and which elicit the immune response. Some cells carry antigens with a wider range of specificities than others, and the density of antigenic representation may also vary from cell to cell. Some leucocytes are highly immunogenic, and Guttmann et $a l^{1}$ believe that this immunogenicity is of major importance in rejection reactions. In an attempt to destroy the so-called immunogenic passenger leucocytes that lodge in the graft they have therefore given kidney graft donors intensive cytotoxic therapy before removal of the kidneys. Their pretreatment consisted of enormous doses of cyclophosphamide and prednisone, 4-6 g of each drug, the cyclophosphamide being given 5-6 hours before removing the kidneys.

If Guttmann et al are right, a kidney devoid of passenger leucocytes would be expected to elicit an immune reaction of lower intensity than a conventionally removed kidney. ${ }^{12}$ They have claimed better results with kidneys removed from pretreated donors, but the numbers were small and the recipients of kidneys from conventional, non-treated donors were not part of a controlled study. Nevertheless, Zincke and Woods ${ }^{3}$ have reported a similar regimen of pretreatment from the Mayo Clinic and obtained similar results. They chose three groups of patients in whom donor and recipient tissue typing and immunosuppressive treatment were thought to be roughly similar. Group A contained 21 patients who received pretreated renal grafts removed from donors in the Mayo Clinic; group B were 23 recipients who received non-treated grafts taken from donors in the Mayo Clinic; and group C, 16 patients, received kidney grafts taken from non-treated donors outside the Mayo Clinic. The two-year actuarial graft survivals were 16 out of 21 in group A, 13 out of 23 in group B, and 7 out of 16 in group C.

The Mayo team concluded that pretreatment of donors significantly improved allograft survival, but most workers would be cautious in accepting these conclusions on so few cases without strict controls. We need more information about possible acute damage to kidneys from hypotension during the pretreatment phase, and these enormous doses of cyclophosphamide and steroids might be expected to harm the kidneys as well as the passenger leucocytes. There is no suggestion of kidney damage in the reports to date, but longterm follow-up of properly controlled series of patients seems to be essential before a case is made for adopting this practice as a routine.

Ethical problems also arise. The donors in both series were patients certified as having brain death, and permission for the pretreatment was obtained from the relatives. Even so, a sense of disquiet and unease might be expected in those caring for the donor if such a procedure were adopted in Britain-unless it were proved beyond all doubt that pretreatment greatly improved results. It is difficult to know where to draw the line in the management of potential donors. Taking blood for tissue typing and maintaining adequate hydration are unlikely to be controversial, since these do not interfere with routine management, but a six-hour pretreatment protocol may be seen as a change in normal care.

1 Guttmann, R D, Beaudoin, J G, and Morehouse, D D, Transplantation Proceedings, 1973, 5, 663 .

2 Guttmann, R D, et al, Transplantation Proceedings, 1975, 7, 117

${ }^{3}$ Zincke, H, and Woods, J E, Surgery, Gynecology, and Obstetrics, 1977, $145,183$.

\section{Government advice on health}

The Ombudsman's report of his inquiry ${ }^{1}$ into vaccination against whooping cough ( $\mathrm{p}$ 1230) and the reactions to it in the national press ${ }^{2} 3$ have raised again the question of the responsibilities of Government for public health and for individual clinical decisions.

No one doubts that the Government (acting on expert advice) has a duty to maintain adequate standards of purity in water supplies, to control food additives, to protect the public against health hazards such as excess lead in petrol or the indiscriminate use of asbestos, and-though here the present Government has failed-to set safe speed limits and to require motorists to wear seat belts.

The Government has also taken on itself the responsibility for controlling the testing of new drugs and is looking at ways of monitoring their use more closely; and here some might argue that the recommendations of the Committee on Safety of Medicines encroached on clinical freedom when they effectively set an upper limit to the oestrogen content of combined oral contraceptives. But the Ombudsman has taken this trend a stage further by suggesting that the Department of Health had a duty to inform the public of the apparent risks and benefits of a specific vaccine. Inevitably this advice was then interpreted and analysed by a whole range of more (or less) expert commentators in the press, radio, and TV. What's wrong with that, many people will ask. Should not the public be better nformed and should doctors not welcome the trend for patients to want a greater say in their choice of treatment ? Certainly. A knowledgeable public is likely to be more health-conscious and will be more receptive to advice on life style, diet, exercise, and smoking. Conflicts are likely only because in much of 